\title{
A FEASIBILITY STUDY: EMOTIONAL FREEDOM TECHNIQUES FOR DEPRESSION IN AUSTRALIAN ADULTS
}

\author{
${ }^{1}$ Peta Stapleton, ${ }^{2}$ Sharon Devine, ${ }^{3}$ Hannah Chatwin, ${ }^{4}$ Brett Porter and ${ }^{5}$ Terri Sheldon \\ ${ }^{1,2,3}$ School of Humanities, Bond University, Gold Coast, Queensland, 4229, Australia \\ ${ }^{4,5}$ The Lakeside Rooms, Robina, Qld
}

Received 2014-06-20; Revised 2014-07-09; Accepted 2014-07-12

\begin{abstract}
The purpose of this study was to investigate the feasibility of using Clinical Emotional Freedom Techniques (EFT) to treat Major Depressive Disorder in an adult population by way of a therapeutic group setting. Adults were assigned to EFT group treatment for a period of eight weeks. Diagnostic assessment was completed immediately pre and post treatment using the Mini International Neuropsychiatric Interview. In addition to this, self-report assessments measuring symptomatic evidence of depression were completed by the participants before the treatment, after the treatment and at three month follow-up. Comparisons with a community group were made at pre and post intervention and three month follow-up. The results indicated a change in diagnosis in each of the participants, with data indicating an overall improvement for the treatment group for depressive symptoms. Study implications and limitations are discussed.
\end{abstract}

Keywords: Depression, Adults, Emotional Freedom Techniques, EFT, Cognitive Behavioural Therapy, CBT

\section{INTRODUCTION}

Approximately $20 \%$ of Australians between the ages of 16-85 experience mental illness in each twelve month period (ABS, 2009). Anxiety, depression and substance use are the most frequently reported disorders and often cooccur, due to anxiety possibly leading to depression, or substances being used to self-medicate. In each 12 month period, of the $20 \%$ of Australians with mental illness, $8.5 \%$ have a minimum of two disorders (ABS, 2009). Moreover, while it has been reported that approximately $45 \%$ will develop mental illness in their lifetime (ABS, 2009), treatment is accessed by less than half of those who experience mental illness (CA, 2010; AIHW, 2007).

Depression is reportedly experienced by one in seven Australians (ABS, 2009). Depression carries the third highest impact of disease (WHO, 2008) including Australia (AIHW, 2007), measured by several factors such as financial burden to the economy and death. Depression has also been reported as the highest source of disability in Australia that does not lead to death (AIHW, 2007). According to the WHO (2008), it is estimated that depression will be ranked as the highest health concern globally by the year 2030 .

Depression has been found to have a high likelihood of recurring throughout the lifetime once previously experienced. According to Bolland and Keller (2002), a single depressive episode will recur within a 12 month period in $22 \%$ of the population. If three or more episodes of depression have been experienced, a $90 \%$ chance of recurrence has been reported (Bolland and Keller, 2002). In addition to this, when depressive episodes recur, the likelihood of suicide becomes higher (Bolland and Keller, 2002).

\subsection{The Diagnostic Features of Major Depressive Disorder}

The Diagnostic and Statistical Manual of Mental Disorders, Fifth edition (DSM-5; APA, 2013) outlines several diagnostic features an individual must meet to be diagnosed with Major Depressive Disorder. The main features of Major Depressive Disorder include a depressed mood experienced almost every day, diminished pleasure and difficulty sleeping. In addition

(c) 2014 Peta Stapleton, Sharon Devine, Hannah Chatwin, Brett Porter and Terri Sheldon. This open access article is distributed under a Creative Commons Attribution (CC-BY) 3.0 license which permits unrestricted use, distribution, and reproduction in any medium, provided the original work is properly credited. DOI: $10.3844 /$ crpsp.2014.19.33 
to this, feelings of worthlessness and difficulty concentrating are frequently experienced. For the individual to meet diagnosis for Major Depressive Disorder, they must have experienced five or more symptoms over a two week period, as listed in the DSM 5 (APA, 2013). These symptoms must have caused distress or impairment in the individuals' life and are not due to substance use or any other medical issue (APA, 2013).

Typical treatments for depression include pharmacological, which has become the most popular form of treatment (Beardslee et al., 2003), psychotherapy, counselling and specific interventions such as Cognitive Behavioural Therapy (CBT; WHO, 2004). CBTis considered a gold standard treatment option and is a structured style of therapy requiring direction from the clinician. It typically addresses dysfunctional emotions, maladaptive behaviours and cognitive processes through behaviour modification and problem solving. CBT is thought to be effective for the treatment of a variety of conditions, including mood, anxiety, personality, eating, substance abuse, tic and psychotic disorders. However, research has found that the skill of the therapist is directly related to the outcomes of CBT (Strunk et al., 2010) and at times clients reportthey have felt under pressure to give particular answers when receiving CBT, or that the therapy was not flexible enough to match client needs (Barnes et al., 2013). While meta-analyses find CBT more effective than psychodynamic therapy and equal to other therapies in treating anxiety and depression (Cuijpers et al., 2008; Tolin, 2010), psychodynamic therapy may provide better long-term outcomes (Shedler, 2010). Regular attendance to therapy sessions may also prove difficult, as researchers have found that consistent attendance by depressed individuals can be limited (Barnes et al., 2003). Reasons for nonattendance have been cited as difficulty dealing with emotional issues and confusion regarding the therapeutic process (Barnes et al., 2003). Therapies such as CBT require a commitment from participants to attend so that client receives adequate treatment. According to Forde et al. (2005), between five and eight treatment sessions has been found to be the optimal number when treating depression. Therefore if clients do not attend regularly, treatment will be ineffective.

The use of alternative treatments by depressed individuals is increasing, with research finding 54\% used some form of complementarys or alternative therapy to treat depression in the United States (Kessler et al., 2001). In addition to this, $66.7 \%$ of those receiving standard treatment for depression, also used alternative therapies (Kessler et al., 2001). However, only $19 \%$ of these with severe depression sought out an alternative therapy provider (Kessler et al., 2001), indicating a preference of for self-administration.

Emotional Freedom Techniques (Clinical EFT) is an approach that uses a tapping technique to heal emotional discomfort (Gallo, 2004). Clinical EFT has been found to be a fast and simple therapy technique, which can be self-administered, or taught by a therapist (Wells et al., 2003). It has been found to be effective for many psychological disorders such as phobias (Jones et al., 2011; Salas et al., 2011; Sezgin and Özcan, 2009; Wells et al., 2003), anxiety (Rowe, 2005), Posttraumatic Stress Disorder (PTSD) (Church, 2008a) and stress (Church, 2008b).

Emotional freedom techniques. Emotional Freedom Techniques (EFT or Clinical EFT) is based on Chinese medicine and the theory of acupuncture, using the energy meridians of the body (Feinstein, 2008). EFT includes elements of cognitive therapy (Beck et al., 1979), exposure (Wolpe, 1969), mindfulness and systematic desensitization (Wolpe, 1958). The process of EFT is completed by becoming aware of a problem, using a setup statement to focus the mind on the issue and tapping on eight specific acupressure points of the body, while verbally stating the issue being addressed. The issue is spoken as a short phrase while tapping. Individuals are initially asked to rate their distress on a subjective units of distress scale from zero to 10 (where zero is no distress and 10 equals the most distress) and after completing a round of each of the tapping points, to re-rate their level of distress. They are then encouraged to continue the rounds until their emotion has reduced.

The procedure of EFT begins by the individual stating a difficulty they are experiencing, followed by an opposing, but positive affirming statement. For example, an individual may state "Even though I am overweight, I deeply and completely love and accept myself". Researchers have found that when positive and negative thoughts are combined, the individual experiences a decrease of the negative experience (Kazdin and Wilcoxon, 1976). This combination of positive affirmation and negative thoughts is typically used in Systematic Desensitization, a behaviour modification therapy (Kazdin and Wilcoxon, 1976).

The practise of Clinical EFT appears to have physiological effects, with one randomised trial measuring cortisol levels related to depression and anxiety, before and after one of three treatment modalities: Clinical EFT, psychotherapy, or relaxation (Church et al., 2012). The findings were that the being 
activated by fear, brain frequencies were found to normalise after treatment (Church et al., 2012).

Brain frequencies have also been found to be affected by Clinical EFT, where after being activated by fear, brain frequencies were found to normalise after treatment with Clinical EFT (Diepold and Goldstein, 2008). Researchers also found when participants were asked to recall a traumatic memory, these frequencies remained in the normal range (Diepold and Goldstein, 2008). The amygdala has been found to be unaffected by stress after immediate use of Clinical EFT (Feinstein, 2008) and the ratio of serotonin and dopamine has been found to balance after an individual has been treated with Clinical EFT (Feinstein et al., 2005).

Neutral plasticity has been noted as a contributing factor to the effects of Clinical EFT (Mollon, 2007). According to Nader et al. (2000), when an individual recalls an emotion inducing image, the memory becomes fluid and further information may be added to the memory. Mollon (2007) states that this is the opposite of avoidance, where memories are shunned and new information cannot be added. When tapping occurs during memory recall, it appears to allow the emotional reaction to be reset, due to this opening of the memory processes.

Clinical EFT has been found to be effective for treating anxiety. In a study that took place over a 14 year period Andrade and Feinstein (2004) measured 5000 individuals, from 11 clinics in South America, being treated for anxiety. The design was double blind and randomised. Two groups were allocated for the study, the control group and the experimental group. The control group was treated with both Cognitive Behavioural Therapy (CBT) and medication, while the experimental group was treated with Clinical EFT. The findings were that $90 \%$ of the symptoms experienced by the Clinical EFT treatment group were reduced and $76 \%$ of the group were reported to no longer experience symptoms of anxiety. The group receiving CBT was found to have a $63 \%$ reduction in symptoms, with $51 \%$ of the group found to no longer experience symptoms. Although both the CBT plus medication and the Clinical EFT treatments were effective, the Clinical EFT group required only three sessions to achieve these results, whereas the CBT and Medication group required 15 sessions. At twelve month follow-up, the CBT group members were found to be more likely to experience relapse than the Clinical EFT group.

Clinical EFT has been found to be effective when treating food cravings in a randomised clinical study (Stapleton et al., 2011). Ninety-six overweight or obese individuals completed a four week treatment program, with participants being measured pre and post the program and also at 6- and 12- month follow-up. Findings indicated a significant improvement in food cravings, the subjective power of food and craving restraint when compared to waitlist controls $(\mathrm{p}<0.05)$ (Stapleton et al., 2011). In addition to this, the improvement in food cravings was maintained at 6and 12- month follow-up, with a craving restraint appearing to have increased effects after time and average weight loss at 12-months was $5.5 \mathrm{~kg}$ (11 pounds) (Stapleton et al., 2011).

\subsection{Clinical EFT and Depression}

Two Randomised Controlled Trials (RCT) found Clinical EFT to be an effective treatment when treating symptoms of depression. After completing an eight week online Clinical EFT course, 36 fibromyalgia patients receiving treatment for PTSD were found to have a significant reduction of depressive symptoms (Brattberg, 2008). The second RCT focussed on 59 war veterans who were experiencing clinical levels of depression (Church et al., 2013). After six sessions of Clinical EFT, there was a significant change in their depressive levels which decreased to a subclinical level. These improvements were also maintained at three and six month follow-up (Church et al., 2013).

A randomised control trial was completed comparing Eye Movement Desensitisation and Reprocessing (EMDR) with EFT in 46 participants (Karatzias et al., 2011). Both of these interventions were found to effectively treat PTSD after four sessions, leading to a full reduction of symptoms (Karatzias et al., 2011). When specifically reviewing the symptoms of depression, both therapies were found to be equally successful, utilising the same amount of time to improve symptoms (Karatzias et al., 2011).

EFT has been found to be effective when treating students with depression. A randomised control trial was conducted on 238 college students with depression, who each participated in four $90 \mathrm{~min}$ group sessions of EFT (Church et al., 2012). When compared to a nontreatment control group, the EFT group had significantly less depressive symptoms than the control group (Church et al., 2012). Limitations to this study include; the EFT sessions were not administered by a trained professional, a small number of sessions were utilised and no formal diagnosis was made of the participants (Church et al., 2012). It was proposed that a larger number of treatment sessions, administered by a trained Clinical EFT professional may provide further effects (Church et al., 2012). 
Clinical EFT has also been found to be an effective treatment when self-administered. A study of 216 health care professionals using Clinical EFT to treat Major Depressive Disorder and anxiety used computer based interventions where the participant was required to follow directions, rather than being taught by a Clinical EFT professional in person (Church and Brooks, 2010). This study found that post treatment, a significant decrease in pains, cravings, emotional instability and traumatic memories was reported ( $\mathrm{p}<0.001)$ (Church and Brooks, 2010).

Large group intervention has also been found to be effective with 102 participants in a three day EFT workshop being measured at the beginning and end of the workshop, as well as at one month and six month follow-up (Rowe, 2005). A significant decrease in symptoms such as anxiety, depression, phobias, somatization, hostility, paranoia and interpersonal sensitivity occurred, as measured by a self-reported symptom checklist (Rowe, 2005). These changes were maintained at three and six month follow-up (Rowe, 2005).

While Clinical EFT has been found to be an effective treatment modality for many disorders (Brattberg, 2008; Church 2008c; Stapleton et al., 2011), its limitations include small sample sizes, lack of professional instruction and lack of formal diagnosis (Church et al., 2012). The benefits of using Clinical EFT include the simplicity of administration (Church and Brooks, 2010) and the reduced financial costs due to the reduced number of treatment sessions required (Wells et al., 2003). Once taught the tapping points and procedure of administration, Clinical EFT can be self-administered. Previous studies have found self-administration of Clinical EFT to be a successful form of therapy (Brattberg, 2008), with effects noted after minimal sessions such as a single session when treating phobias (Wells et al, 2003), or four sessions for successful treatment of PTSD (Church, 2010). Thus, Clinical EFT is a simple therapy, which may be administered at anytime. As depression is a growing concern amongst the population and current therapy is not universally effective, this may be an alternative therapy for those who are unmotivated, fatigued and experiencing financial hardship.

The significance of this study is its exploration of the use of Clinical EFT in the clinical setting for the first time with adults suffering Major Depressive Disorder. Clinical EFT has been shown to be effective in the treatment of stress (Church, 2008b), anxiety (Rowe, 2005), phobias (Jones et al., 2011; Wells et al., 2003) and PTSD (Church, 2010). While symptoms of depression have been found to improve when other psychological conditions have been treated with EFT (Brattberg, 2008; Church et al., 2011), no study has measured the effect of EFT solely on Major Depressive Disorder in an adult population, using a diagnostic tool as evidence for treatment effects. The current study aimed to develop an eight week Clinical EFT program and test its effectiveness in treating adults with diagnosed Major Depressive Disorder. Each of the 120 minute Clinical EFT treatment sessions would be conducted by a trained Clinical EFT professional. A formal DSM-IV (APA, 1994) diagnosis of Major Depressive Disorder would be assessed prior to the commencement of treatment and reassessed at the conclusion of treatment. Selfreported symptomatic levels of depression and any comorbid conditions would be measured at the commencement of treatment and again at the conclusion of treatment and three month follow-up.

It was hypothesised that Clinical EFT would prove to be a successful treatment modality in this feasibility study for reducing Major Depressive Disorder in an adult sample, as measured by the Mini International Neuropsychiatric Interview (MINI; Sheehan et al., 1998), Beck Depression Inventory II (BDI-II; Beck et al., 1961) and the Depression, Anxiety and Stress Scale 21(DASS 21; Lovibond et al., 1993). It was also hypothesised that Clinical EFT would have sustained benefits at the 3month follow-up period.

\section{MATERIALS AND METHODS}

\subsection{Participants}

Eleven adults were recruited using Face book pages, university notice boards, word of mouth and community classified pages in both local newspapers and on the internet. The criteria to participate in the trial included being over 18 years of age and having a diagnosis or probable diagnosis of Major Depressive Disorder. Volunteer participants were required to contact the researcher and an appointment was made to complete the MINI (Sheehan et al., 1998). The MINI was used to confirm diagnosis of Major Depressive Disorder. Participants' were given the option to either meet with the researcher to complete the MINI at the University where the study was held, or complete the MINI over the telephone. Each adult was emailed contact details of alternative services, such as Lifeline, to be used if they became distressed by completing the MINI, or if they did not meet criteria for Major Depressive Disorder. 
After completing the MINI, it was found that ten of the eleven participants met criteria for Major Depressive Disorder (additional diagnoses can be viewed in Table 5). The additional participant did not meet diagnostic criteria for either Major Depressive Disorder, or an alternate disorder, however, was accepted into the treatment group due to self- reported depressive symptoms. Once the participants had met diagnostic criteria for Major Depressive Disorder, they completed an online questionnaire package including demographic questions such as age range, gender, education level, number of people in household, current education level, as well as the Beck Depression Inventory II (BDI-II; Beck et al., 1996) and the Depression, Anxiety and Stress Scale 21(DASS 21; Lovibond et al., 1993).

The final depressed sample consisted of two males and nine females who participated voluntarily after providing informed consent (see Table 1). Of the males, one was in the 25-29 year old age range and one was in the over 55 age range. Of the female participants, three were in the 18-24 year old age range, one was in the 3035 year old age range, two were in the 40-45 year old age range, one was in the 46-49 year old age range and two were in the 50 and over age range.

A comparison community sample of non-depressed adults was recruited through Facebook pages, university noticeboards and community classified pages in both local newspapers and on the internet. The community sample consisted of 34 individuals, sevenmales and 27 females each of whom participated voluntarily after providing informed consent (Table 2). Of the males, one was in the 30-35 year old age range, one was in the 40-45 year old age range and five were in the $50+$ age range. Of the female participants, two were in the 18-24 year old age range, one was in the 25-29 year old age range, three were in the 30-45 year old age range, two were in the 36-39 year old age range, eight were in the 40-45 year old age range, three were in the 46-49 year old age range and eight were in the 50 and over age range.

\subsection{Intervention and Materials}

This study was reviewed for human subject protection by the Bond University Research Ethics Committee (BUHREC). Protocol Number: RO1518 and was registered with The Australian New Zealand Clinical Trials Registry (ANZCTR).
The Clinical EFT instruction was provided by a certified professional EFT practitioner who acted as group facilitator and a student researcher acted as a support facilitator. The group intervention took place at the Bond University Psychology Clinic.

The Clinical EFT program was developed by the supervising and student researcher and two certified EFT therapists, was based on standard Clinical EFT protocols as outlined in the EFT manual (Craig, 2010) and was then peer reviewed. In addition, this program was modelled on a previous EFT food craving program developed by the supervising researcher (Stapleton et al., 2011). The program included a treatment fidelity plan, requiring completion of an intervention checklist after each treatment group by the treatment professional and the co-facilitator.

The EFT program was designed to increase participants' understanding of Major Depressive Disorder, as well as provide instruction regarding thoughts and their effects on feelings, the benefits of increased activity levels, psycho-education regarding EFT and its associated tapping points, how to construct appropriate EFT statements and to experience the effects of using EFT.

The process of Clinical EFT involves thinking of adistressing event, emotion, or memory, ratings its intensity, initiating a setup statement, then completing the tapping process on a sequence of 7 specific points on the upper body (Brattberg, 2008). The setup phrase focuses on the difficulty being experienced and is spoken aloud, adding a voiced statement of acceptance. For example, if anxiety is experienced, a setup phrase could be "Even though I have this anxiety, I deeply and completely accept myself." The setup phrase is stated while tapping either the "karate chop" point below the little finger of either hand, or a specific area on the chest below the collar bone, termed the sore spot in EFT protocols (Craig, 2010). After repeating the setup phrase three times, the tapping process is followed, using a shortened version of the setup phrase. In the example above a phrase such as "All this anxiety" would be appropriate and would be voiced while each point is tapped approximately seven times each. Acupressure points 1 to 7 (Craig and Fowlie, 1995) were taught to each participant to use when self-administering EFT. These points are the inner eyebrow, the side of the eye, under the eye, under the nose, the chin, the collar bone and under the arm. See diagram in Appendix A. 
Table 1. Age range and sex distribution of depressed sample

\begin{tabular}{llllllll}
\hline Age & $18-24$ & $25-29$ & $30-35$ & $36-39$ & $40-45$ & $46-49$ & $50+$ \\
\hline Male & 0 & 1 & 0 & 0 & 0 & 0 & 1 \\
Female & 3 & 1 & 0 & 0 & 2 & 1 & 2 \\
\hline
\end{tabular}

Table 2. Age range and sex distribution of community sample

\begin{tabular}{llllllll}
\hline Age & $18-24$ & $25-29$ & $30-35$ & $36-39$ & $40-45$ & $46-49$ & $50+$ \\
\hline Male & 0 & 0 & 1 & 0 & 1 & 0 & 5 \\
Female & 2 & 1 & 3 & 2 & 8 & 3 & 8 \\
\hline
\end{tabular}

Two main types of instructional materials were used during the intervention; instructional material administered in the form of PowerPoint slides and client handouts developed by the lead author were handed out at the beginning of each session. The session topics for the EFT intervention consisted of the following:

Week 1: Psychoeducation regarding treatment

Week 2: Behaviours that help manage depression

Week 3: The Thinking-Feeling connection

Week 4: Cognitive Restructuring

Week 5: Core Beliefs

Week 6: Stress and Relaxation

Week 7: Goal Getting

Week 8: Self Management

Social validity data was gathered in each group on a weekly basis, by way of participants completing self-report measures regarding the effectiveness of treatment. Participants were able to remain anonymous in order to obtain the most accurate feedback. The participants were asked to nominate on a Likert scale from 1 to 6 , with 1 indicating not useful at all and 6 indicating very useful, how useful the treatment information and skills learnt were to their symptoms of depression. Participants were then asked to nominate how easy the treatment information was to understand, with 1 indicating very difficult and 6 indicating very easy. Participants were also asked to indicate how confident they felt in using the information and skills covered, with 0 indicating not confident at all and 6 indicating confident. Finally, participants were asked if they would recommend using these new skills to others, with 1 indicating not recommended and 10 indicating highly recommended.

Participants were also asked to provide a rating regarding the facilitator, with 1 indicating not at all and 5 indicating very much. Participants were asked how approachable the facilitator was, how well the facilitator explained skills and ideas, how well questions and concerns were addressed, how appropriate handout materials were and how much the program met their needs. They were also invited to make comments at the end of the form.

The current study was a pilot study using a small sample of 11 participants, being compared to a nonclinical community sample.

\subsection{Measures}

The Mini International Neuropsychiatric Interview (MINI; Sheehan et al., 1998) is a short structured clinical interview, based on the Structured Clinical Interview for DSM IV (SCID; First et al., 1997) which enables researchers to make diagnoses of psychiatric disorders according to DSM-IV or ICD-10. Taking approximately $15 \mathrm{~min}$ to complete, the MINI has been found to have good validity and reliability (Sheehan et al., 1998). Sheehan et al. (1997) indicated the MINI has been found to have a high correlation with the Structured Clinical Interview for DSM Axis I Disorders, as well as having both strong sensitivity (0.96) and specificity (0.96).

The Beck Depression Inventory II (BDI; Beck et al., 1996) was used to assess symptom severity in clients with probable Major Depressive Disorder. Each item contained a statement which the client then rates in relation to themselves, with higher total ratings indicating higher levels of depression. Total scores less than 10 are considered to reflect no or minimal depression, while scores ranging between 10 and 18 are considered to reflect mild depression. Moderate depression is indicated by scores between 19 and 29, while severe depression is indicated by scores greater than 29. The BDI II has convergent validity with observer-rated measures diagnosing depression (Marton et al., 1989). Internal consistency of the BDI-II has been shown to be consistently high, with measures of Cronbach's alpha ranging from.88-0.95(Beck et al., 1996).

The Depression, Anxiety, Stress Scale 21 (DASS 21; Lovibond and Lovibond, 1995) is a self-report questionnaire consisting of 21 items, which provides a measure of depression, anxiety and stress (Atkin and Cetin, 2007). The DASS 21 has been found to have 
good internal consistency, with interscale correlations being Depression-Anxiety (0.45-0.71), 0.50 or below in all English-speaking samples, Anxiety-Stress (0.65-0.73) and Major Depressive Disorder-Stress (0.57-0.79) (Antony et al., 1998; Brown et al., 1997; Clara et al., 2001). Convergent validity has also been found to be good for both clinical and non clinical samples (Brown et al., 1997).

\subsection{Participant Flow}

Twenty individuals expressed interest in the program. Of these individuals, 13 agreed to be assessed (Fig. 1). Two participants did not attend assessment, however, each of the 11 participants who were assessed, participated in the treatment program. Of those members, 2 males and 3 females did not return after 2 weeks due to several factors. Two withdrew due to illness, 2 had a lack of confidence in the program and 1 participant did not return due to death in the family and resultant grief (Fig. 1). Of the remaining members $(n=6)$, each completed the final assessments and 3-month follow-up. The withdrawn participants did not appear to differ from completers by visual inspection of pre-treatment scores or demographic characteristics.

Eight Clinical EFT group therapy sessions were administered over eight consecutive weeks. Each session lasted 120 min. Participants were not included in the data analysis if they discontinued attendance after the first two weeks $(n=5)$. Treatment was provided through a group setting, with information being presented by the certified EFT professional, using the PowerPoint slides as a treatment tool, as well as modelling tapping points, as previously described. Participants were given handouts, pens and a clipboard and encouraged to stop and complete areas in the handouts regarding symptoms, thoughts and feelings as needed.

Participants who provided consent to do so, were sent text messages on a weekly basis by the certified EFT therapist, reminding them to use their Clinical EFT techniques daily, which was designed to strengthen motivation and adherence. Participants were able to withdraw from this service at any time and were asked periodically throughout the study if the frequency of contact was appropriate and if there were any concerns regarding this practise.

The post-test was administered at the conclusion of the final session. Three month follow-up was completed by an email being sent to each participant, containing a link to the measures.

\subsection{Data Analysis}

Data was analysed using the Statistical Package for Social Sciences 22.0 (SPSS). An independent samples t-test was conducted to compare the self-reported baseline DASS 21 scores between the depressed and the non-depressed group. There was significant difference in scores between the depressed $(\mathrm{M}=28.66, \mathrm{SD}=9.41)$ and the nondepressed group $(\mathrm{M}=6.11, \mathrm{SD}=4.63$; $\mathrm{t}(38)=9.25, \mathrm{p}=$ 0.00 (two-tailed). The magnitude of the differences in the means (mean difference $=22.54,95 \%$ CI: $17.61-27.48$ was large (eta squared $=0.69$ ).

An independent samples t-test was conducted to compare the self-reported DASS 21 scores at the completion of treatment between the depressed and the non-depressed group. There was a significant difference in scores between the depressed $(\mathrm{M}=28.16, \mathrm{SD}=14.56)$ and the non-depressed group $\mathrm{M}=6.11, \mathrm{SD}=4.63 ; \mathrm{t}$ (38) $=3.67, \mathrm{p}=0.01$ (two-tailed). The magnitude of the differences in the means (mean difference $=22.04,95 \%$ CI: 6.78-37.31 was large (eta squared 0.26).

An independent samples t-test was conducted to compare the self-reported DASS 21 scores at three month follow-up between the treatment group and the community sample. There was significant difference in scores between the depressed $(M=21.16, S D=9.49)$ and the non-depressed group $(\mathrm{M}=6.11, \mathrm{SD}=4.63 ; \mathrm{t}$ $(5.42)=3.80, p=0.01)$. The magnitude of the differences in the means (mean difference $=15.04,95 \%$ CI: 5.11 to 24.98 ) was large (eta squared $=0.27$ ).

An independent samples t-test was also conducted to compare the self-reported baseline BDI II scores between the depressed and non depressed group. There was a significant difference between the depressed $(\mathrm{M}=$ $30.66, \mathrm{SD}=11.00)$ and the non-depressed group $(\mathrm{M}=$ $5.08, \mathrm{SD}=5.41 ; \mathrm{t}(38)=8.97, \mathrm{p}=0.00$ (two tailed). The magnitude of the differences in the means (mean difference $=25.57,95 \%$ CI: 19.80 to 31.34 ) was large (eta squared $=0.67$ ).

An independent samples t-test was conducted to compare the self reported post BDI II scores between the depressed and non-depressed group. There remained a significant difference between the depressed $(\mathrm{M}=21.83, \mathrm{SD}=14.86)$ and the nondepressed group $(\mathrm{M}=5.08, \mathrm{SD}=5.41, \mathrm{t}(5.23)=2.72$, $\mathrm{p}=0.039$ (two tailed). The magnitude of the differences in the means (mean difference $=16.74$, 95\% CI: 1.17 to 32.31 ) was large (eta squared $=0.16$ ). 
Peta Stapleton et al. / Current Research in Psychology 5 (1): 19-33, 2014

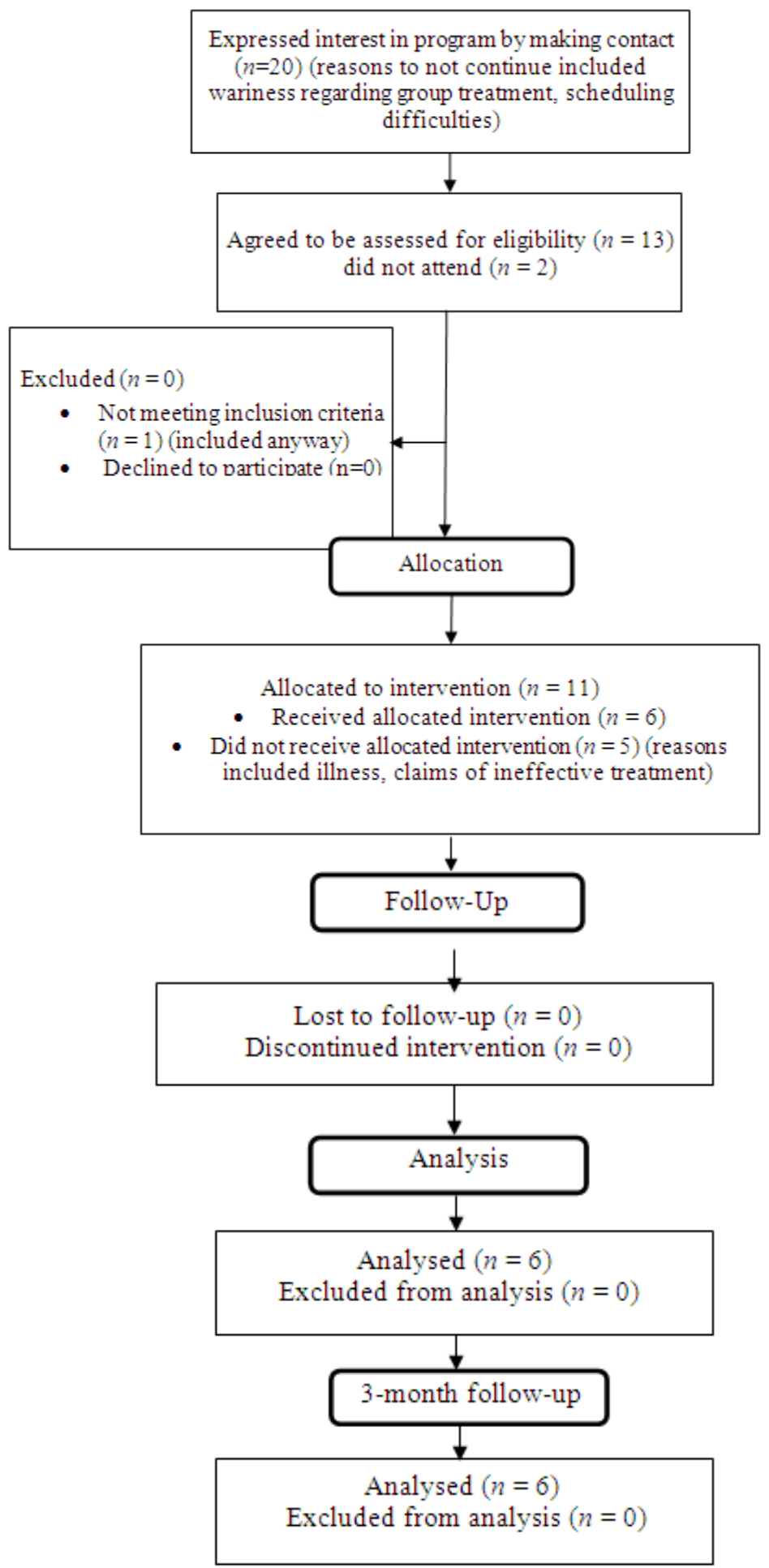

Fig. 1. Participant flow diagram of clinical EFT treatment group 
Table 3. Descriptive statistics for DASS 21 administration Time 1, Time 2 and Time 3

\begin{tabular}{llrr}
\hline Time Period & N & Mean & Standard deviation \\
\hline Time 1 (Pre-intervention) & 6 & 28.66 & 9.42 \\
Time 2 (Post intervention) & 6 & 28.16 & 14.56 \\
Time 3 (3 month follow-up) & 6 & 21.16 & 9.50 \\
\hline
\end{tabular}

Table 4. Descriptive Statistics for BDI II for Time 1, Time 2 and Time 3

\begin{tabular}{lllr}
\hline Time Period & $\mathrm{N}$ & Mean & Standard deviation \\
\hline Time 1 (Pre-intervention) & 6 & 30.66 & 4.49 \\
Time 2 (Post intervention) & 6 & 21.83 & 6.07 \\
Time 3 (3 month follow-up) & 6 & 18.66 & 7.20 \\
\hline
\end{tabular}

An independent samples t-test was conducted to compare the self-reported BDI II scores at three month follow-up between the treatment group and the community sample. There was a significant difference in scores between the depressed $(\mathrm{M}=18.66, \mathrm{SD}=$ $10.93)$ and the non-depressed group $(\mathrm{M}=5.08, \mathrm{SD}=$ $5.41 ; \mathrm{t}(5.44)=2.97, \mathrm{p}=0.028)$. The magnitude of differences in the means (mean difference 13.57, 95\% CI: 2.14 to 25.01 ) was large (eta squared $=0.18$ ).

The means and standard deviations of the administration of the DASS 21 and pre treatment, post treatment and three month follow-up are presented in Table 3.

The means and standard deviations of the administration of the BDI II, at pre intervention, post intervention and three month follow-up are indicated in Table 4.

A Wilcoxon Signed Rank Test revealed no significant reduction in symptoms as measured by the DASS 21 when comparing the pretest scores and the 3month follow-up, $\mathrm{z}=-1.68, \mathrm{p}=0.093$. However, $\mathrm{a}$ Wilcoxon Signed Rank Test revealed a significant reduction in depressive symptoms as measured by the BDI II when comparing the pretest scores and the 3month follow-up, $\mathrm{z}=-2.20, \mathrm{p}=0.028$.

\section{RESULTS}

\subsection{EFT Treatment}

Eleven individuals were recruited through university notice boards, Facebook pages and word of mouth. Ten of these individuals met criteria for Major Depressive Disorder, which was assessed by the administration of the MINI. One participant did not meet criteria for Major Depressive Disorder, however, participated in the group due to depressive symptomology.

The first hypothesis asked whether Clinical EFT resolved Major Depressive Disorder as a diagnosis. The data revealed that while the diagnosis of Major Depressive Disorder was not completely resolved immediately after treatment, two members no longer met criteria. In addition, each member of the group no longer met diagnosis for one or more disorders they had met criteria for when completing the MINI.

The second hypothesis asked whether Clinical EFT was effective at reducing the symptoms of Major Depressive Disorder. While scores on the DASS 21 indicated no significant difference, in several cases individual participants reported clinical differences in symptoms. That is, the difference was enough so that if measured in a clinical setting, it would indicate treatment was successful. Therefore a clinically valid difference was achieved. The BDI II indicated a significant difference in the pre, post and 3-month follow-up measures in the symptoms of Major Depressive Disorder.

The third hypothesis asked whether the treatment effects of Clinical EFT were sustained at follow-up. The data suggests that not only are the effects of Clinical EFT sustained at follow-up, but there appeared to be continuous effects.

\subsection{Participant Evaluation and Subjective Ratings}

Each participant in the study was asked to complete a worksheet containing social validity questions at the end of each session. This was on a voluntary basis and could be completed anonymously. The answers provided indicated that throughout the program $88 \%$ found the Clinical EFT program information and skills to be useful. In addition, $100 \%$ found the information easy to understand and $88 \%$ indicated they felteither okay, confident, or higher in using the information and skills covered.

Feedback regarding the facilitator was given using a Likert scale from 1-5, with 1 indicating Not at all and 5 indicating Very much. When asked how approachable the facilitator was $4 \%$ indicated a score of 4 , whereas $96 \%$ gave the maximum score of 5 . When asked how well the facilitator explained skills and ideas, 24\% indicated a score of 4 , while the remainder of the participants gave a score of 5 . When asked how well the facilitator addressed questions and concerns, $17 \%$ gave a score of 4 , whereas $83 \%$ gave a score of 5 . When asked how appropriate the handout materials were, $2 \%$ gave a 
score of 3,33\% gave a score of 4, with $65 \%$ giving a score of 5. The final question asked at what level the program met the individuals' goals. $7 \%$ gave a score of $3,30 \%$ gave a score of 4 and $63 \%$ gave a score of 5 .

Each participant was re-administered the MINI at the completion of the program. As indicated in Table 5, of all EFT the participants, both participant 2 and 5 no longer met criteria for Major Depressive Disorder (MDD). Participant 1 maintained the diagnosis of MDD and General Anxiety Disorder (GAD), however, no longer met criteria for Agoraphobia, Social Anxiety Disorder (SAD) and Obsessive Compulsive Disorder (OCD). Participant 4 maintained the diagnosis for MDD, however no longer met criteria for GAD. Both participant 5 and participant 2 attended the entire eight week program and had the greatest results. Participant 5 no longer met criteria for MDD, Panic Disorder with Agoraphobia, OCD Current, or GAD. Participant 2 no longer met criteria for MDD, Panic Disorder and Bulimia Nervosa. At the initial administration, participant 3 met criteria for MDD, Panic Disorder and Agoraphobia, SAD, OCD, PTSD, Bulimia and GAD, whereas, when the interview was readministered, the participant no longer met criteria for OCD.

Although the changes in DASS 21 scores were not significant, some changes were at a level to be clinically remarkable and to have indicated change at a clinical level (Table 6).

Table 5. EFT Pre and post diagnosis from structured clinical interview

\begin{tabular}{|c|c|c|}
\hline Participant & Pre Diagnosis & Post diagnosis \\
\hline 1 & $\begin{array}{l}\text { Major depressive disorder } \\
\text { Agoraphobia } \\
\text { Social anxiety disorder } \\
\text { Obsessive compulsive disorder } \\
\text { General anxiety disorder }\end{array}$ & $\begin{array}{l}\text { Major depressive disorder } \\
\text { General anxiety disorder }\end{array}$ \\
\hline 2 & $\begin{array}{l}\text { Major depressive disorder } \\
\text { Panic disorder } \\
\text { Bulimia nervosa }\end{array}$ & No diagnosis met \\
\hline 3 & $\begin{array}{l}\text { Major depressive disorder } \\
\text { Panic disorder } \\
\text { Social anxiety disorder } \\
\text { Obsessive compulsive disorder } \\
\text { Post traumatic stress disorder } \\
\text { Bulimia nervosa } \\
\text { General anxiety disorder }\end{array}$ & $\begin{array}{l}\text { Major depressive disorder } \\
\text { Panic disorder } \\
\text { Social Anxiety Disorder } \\
\text { Post traumatic stress disorder } \\
\text { Bulimia nervosa } \\
\text { General Anxiety disorder }\end{array}$ \\
\hline 4 & $\begin{array}{l}\text { Major depressive disorder } \\
\text { Generalised anxiety disorder }\end{array}$ & Major depressive disorder \\
\hline 5 & $\begin{array}{l}\text { Major depressive disorder } \\
\text { Panic disorder } \\
\text { Obsessive compulsive disorder } \\
\text { General anxiety disorder }\end{array}$ & No diagnosis \\
\hline
\end{tabular}

Table 6. The depression scale of the DASS 21

\begin{tabular}{llll}
\hline Participant & DASS 21 pre & DASS 21 post & DASS 21 3mth follow-up \\
\hline 1 & 15 & 20 & 14 \\
& Extremely severe & Extremely Severe & Extremely severe \\
2 & 5 & 4 & 4 \\
& Mild & Normal & Normal \\
3 & 15 & 4 & 9 \\
& Extremely severe & Normal & Moderate \\
4 & 15 & 12 & 9 \\
& Extremely severe & Severe & Moderate \\
5 & 8 & 7 & 6 \\
6 & Moderate & Moderate & Mild \\
& 14 & 20 & 17 \\
& Extremely severe & Extremely severe & Extremely severe \\
\hline
\end{tabular}


Table 7. The anxiety scale of the DASS 21

\begin{tabular}{|c|c|c|c|}
\hline & Dass 21 & Dass 21 & Dass 21 \\
\hline Participant & Anxiety pre & Anxiety post & Anxiety 3 mthfollow-up \\
\hline \multirow{2}{*}{1} & 5 & 10 & 9 \\
\hline & Mild & Extremely severe & Severe \\
\hline \multirow[t]{2}{*}{2} & 1 & 0 & 0 \\
\hline & Normal & Normal & Normal \\
\hline \multirow[t]{2}{*}{3} & 5 & 3 & 3 \\
\hline & Mild & Normal & Normal \\
\hline \multirow[t]{2}{*}{4} & 12 & 8 & 5 \\
\hline & Extremely severe & Severe & Mild \\
\hline \multirow[t]{2}{*}{5} & 11 & 9 & 0 \\
\hline & Extremely severe & Severe & Normal \\
\hline \multirow[t]{2}{*}{6} & 6 & 7 & 6 \\
\hline & Mild & Moderate & Mild \\
\hline
\end{tabular}

Table 8. The stress scale of the DASS 21

\begin{tabular}{llll}
\hline Participant & DASS 21 & DASS 21 & DASS 21 \\
\hline \multirow{2}{*}{1} & Stress pre & Stress post & Stress 3 mthfollow-up \\
& 16 & 18 & 11 \\
2 & Severe & Extremely severe & Moderate \\
& 6 & 8 & 7 \\
3 & Normal & Mild & Normal \\
& 7 & 4 & 5 \\
4 & Normal & Normal & Normal \\
5 & 12 & 12 & 9 \\
& Moderate & Moderate & Mild \\
6 & 11 & 12 & 6 \\
& Moderate & Moderate & Normal \\
\hline
\end{tabular}

The symptoms of anxiety as measured by the DASS21 generally continued to reduce after treatment with Clinical EFT had concluded (Table 7). The Stress scale of the DASS 21 indicated a mild improvement at a clinical level in some cases (Table 8).

\section{DISCUSSION}

The current study was a feasibility pilot study of a Clinical EFT program that was developed to treat Major Depressive Disorder in an adult population. The results confirmed that Clinical EFT is an effective treatment modality when treating symptoms of depression. Firstly, each participant treated with Clinical EFT found their depressive symptoms were reduced at some level. This is consistent with previous reports of reduced depression when using Clinical EFT as a primary treatment modality (Church et al., 2012; Feinstein, 2008).

The measures used to assess the symptoms of depression produced differing results. The BDI II indicated a significant difference between the three administrations, whereas the DASS 21 indicated there was no significant difference. The second administration of DASS 21 post-treatment, indicated many of the participants had a greater number of symptoms, or experienced greater severity of their symptoms, than was experienced pre-treatment. There may be several reasons for this. As both post measures question how the participants felt over the past week, this may change on a week to week basis. While there may be an improvement in one week, this may change to an increase in symptoms in an alternate week. Moreover, the second administration of both the DASS 21 and the BDI II, were completed at the conclusion of the final EFT session, while the participants were still in the treatment room. Clinical EFT, by its nature brings into consciousness any negative thought or feeling the participant had been experiencing and as this second administration was completed immediately after a treatment session, it could be hypothesised that each individual was more focussed on their difficulties from the prior week, than they would have been when initially completing the survey. Therefore it may have 
been that an awareness of depressive symptoms had increased throughout the course of treatment, which led to an increase of reporting, over what was initiated in the initial assessment. However, this does not explain the results of the BDI II.

The BDI II administered immediately post-treatment provided an overall indication that participants experienced less symptoms, at a lower severity level, than had been indicated pre-treatment. The variance in the results of the DASS-21 and the BDI II may have been due to studies on the DASS-21 which have found that rather than the depression, anxiety and stress scales measuring symptoms in those specific areas, many of the items appeared to be measuring general distress (Osman et al., 2012). Osman et al. (2012) have suggested that several items of the DASS do not fit specifically in the anxiety, stress or depression scale, leaving the researchers to state that the scales of the DASS 21 appear unreliable. Therefore, the results of the DASS 21 appear to be questionable in a study specifically measuring the symptoms of depression.

The results taken at 3-month follow-up of both the BDI II and the DASS 21 indicated there may have been a progressive improvement effect. Many of the group members experienced a continuous improvement in their symptoms. As previously noted, members of the group were sent periodic supportive text messages to remind them to continue tappingduring the treatment program, although it is uncertain how many members completed this exercise, or how often it was completed. However, if it was ascertained that the gradual improvement occurred without further intervention, this would make it a very powerful treatment modality. A delayed effect was also found by Stapleton et al. (2011), where EFT used to treat food cravings appeared to have continuous effects, with feelings of restraint over food having been found to increase at 6-month follow-up. Alternatively, if these effects were due to the supportive messages, they further indicate the effectiveness of self-administered Clinical EFT.

There were several limitations to this study. The large number of drop outs from the group meant that it effectively halved in size after recruitment. In addition, recruitment proved difficult with a depressed population. While many enquiries were made regarding the program, the group modality of treatment appeared to be a negative factor for enrollment as many initial enquirers stated they would not feel comfortable attending a group setting.

Completing the structured interview provided a difficulty for some members who failed to attend their appointment to complete the interview. Of the participants that did complete the study, difficulty was experienced in receiving completed follow-up questionnaires. As depressed individuals usually have difficulty with motivation and concentration, this is an aspect that would need to be considered for future studies.

\section{CONCLUSION}

Three major conclusions can be made from this study. The first conclusion is that Clinical EFT as a treatment modality appears to resolve symptoms a client is experiencing, which may in turn resolve a disorder that has been diagnosed. In the current study, each participant was asked to practise Clinical EFT on any difficulties they were experiencing over the week and were reminded of this through text messages sent by the Clinical EFT professional on a weekly basis. Although the program was targeted toward depressed individuals, they were encouraged to used Clinical EFT to deal with any issues, which could range from self-esteem issues to anger, difficulties with their partner, or any other difficulties that were experienced. This may account for the change in diagnosis for each participant.

The second conclusion was that Clinical EFT appeared to reduce depressive symptoms in each participant. Although there were no significant differences in the self-report measures of the DASS 21 , the self-report measures of the BDI II indicated a significant difference. The MINI also, reported a change in diagnosis of Major Depressive Disorder in several participants and a change or reduction in the number of comorbid disorders in other participants.

Each participant indicated to the researcher that they felt better and visually appeared to have an altered demeanour and outlook as the sessions progressed. It could be concluded that as improvements were made between the final session and the 3-month follow-up, changes were in a transient state and that 6-month follow-up measures could ascertain whether there is a stronger effect in this area.

The third conclusion was the results were not only sustained at follow-up, but appeared to improve. In addition, participants' appeared to require the full eight weeks of treatment to no longer meet criteria for Major Depressive Disorder. As both participants who attended the entire eight week program no longer met criteria for Major Depressive Disorder it appears that attendance for shorter amounts of time, although effective, did not produce the same results. Further consideration may be that each participant met criteria for several disorders, therefore, as each participant experienced resolution from one of these, they may have required further 
treatment sessions to work through each of their diagnoses to reach the cause of their difficulties and resolve their Major Depressive Disorder.

Future studies could complete longitudinal follow-up assessments on participants to ascertain if the treatment method has long term effects. In addition, due to many enquirers stating they found the group aspect of the treatment daunting, future treatment could focus more on individual treatment on a one on one basis. The current study lends support for a larger trial. Future studies could use more comparison treatment when treating depression, such as relaxation and CBT, as well as utilising a larger sample size.

\section{REFERENCES}

ABS, 2009. Australian Bureau of Statistics National Survey of Mental Health and Wellbeing: Summary of Results, 4326.0, 2007. ABS: Canberra.

AIHW, 2007. The Burden of Disease and Injury in Australia. AIHW: Canberra.

Andrade, J. and D. Feinstein, 2004. Energy Psychology: Theory, Indications, Evidence. In: Energy Psychology Interactive, Feinstein, D. (Ed.), Ashland: Innersource, pp: 199-214.

Antony, M., P.J. Bieling, B.J. Cox, M.W. Enns and R.P. Swinson, 1998. Psychometric properties of the 42item and 21-item versions of the depression anxiety stress scales in clinical groups and community a sample. Psychol. Assessment, 10: 176-181. DOI: 10.1037/1040-3590.10.2.176

APA, 1994. Diagnostic and Statistical Manual of Mental Disorders. 4th Edn., Washington, DC.

APA, 2013. Diagnostic and Statistical Manual of Mental Disorders. 5th Eds., American Psychiatric Publishing, Inc., Arlington, VA.

Atkin, A. and B. Cetin, 2007. The Depression, Anxiety and Stress Scale (DASS): The study of validity and reliability. Educ. Sci.: Theory Practise, 7: 260-268.

Barnes, W.L., A. Dereux and T.W. Ebbesen, 2003. Surface plasmon subwavelength optics. Nature, 424 : 824-830. DOI: 10.1038/nature01937

Barnes, M., S. Sherlock, L. Thomas, D. Kessler and W. Kuyken et al., 2013. No pain, no gain: Depressed clients' experiences of cognitive behavioural therapy. British J. Clin. Psychol., 52: 347-364. DOI: 10.1111/bjc. 12021

Beardslee, W.R., T.R. Gladstone, E.J. Wright and A.B. Cooper, 2003. A family based approach to the prevention of depressive symptoms in children at risk: evidence of parental and child change. Pediatrics, 112: 119-131. DOI: 10.1542/peds.112.2.e119
Beck, A.T., A.J. Rush, B.F. Shaw and G. Emery, 1979. Cognitive Therapy of Depression. 1st Edn., Guilford, New York.

Beck, A.T., C.H. Ward, M. Mendelson, J. Mock and J. Erbaugh 1961. An inventory for measuring depression. Arch Gen. Psychiatry, 4: 561-571. DOI: 10.1001/archpsyc.1961.01710120031004

Beck, A.T., R.A. Steer and G.K. Brown, 1996. Beck Depression Inventory Manual. 2nd Edn., Psychological Corporation, San Antonio TX.

Bolland, R.J. and M.B. Keller, 2002. Course and Outcome of Depression. In: The Handbook of Depression, Gotlib, I.H. and C.L. Hammen (Eds.), The Guilford Press, NY, pp: 43-60.

Brattberg, G., 2008. Self administered clinical EFT in individuals with fibromyalgia: A randomised trial. Integrative Med. Clin. J., 7: 30-35.

Brown, T.A., B.F. Chorpita, W. Korotitsch and D.H. Barlow, 1997. Psychometric properties of the Depression Anxiety Stress Scales (DASS) in clinical samples. Behav. Res. Therapy, 35: 79-89. DOI: 10.1016/s0005-7967(96)00068-X

CA, 2010. National Mental Health Report 2010. Canberra, Australia.

Church, 2008a. Exhibiting as Niquity: Travels of an Acidental Curator. In: Handbook of the Arts in Qualitative Social Science Rearch, A. Cole and J.G. Knowles (Eds.)., Saga, Thousand Oaks. CA, pp: 421-434

Church, 2008b. The Turbulence of Learning to Publish. In: Learning Through Community: Exploring Participatory Practices, K. Church, N. Bascia and E. Shragge (Eds.)., Springer Science and Business Media, B.V., Amsterdam, pp: 5-7.

Church, D., 2008c, The effect of energy psychology on athletic performance: A randomized controlled blind trial. Association for Comprehensive Energy Psychology (ACEP).

Church, D. and A.J. Brooks, 2010. The effect of a brief EFT (Emotional Freedom Techniques) self-intervention on anxiety, depression, pain and cravings in healthcare workers. Integrative Med. Clinician's J., 9: 40-44.

Church, D., 2010. The treatment of combat trauma in veterans using clinical Emotional Freedom Techniques (EFT): A pilot protocol. Traumatology, 16: 55-65. DOI: $10.1177 / 1534765609347549$

Church, D., M. De Asis and A. Brooks, 2012. Brief group intervention using clinical Emotional Freedom Techniques (EFT) for depression in college students: A randomised controlled trial. Major Depressive Disorder Res. Treatment. DOI: $10.1155 / 2012 / 257172$ 
Church, D., O. Pina, C. Reategui and A. Brooks, 2011. Singlesession reduction of the intensity of traumatic memories in abused adolescents after EFT: A randomized controlled pilot study. Traumatology, 18: 73-79. DOI: $10.1177 / 1534765611426788$

Church, D., C. Hawk, A.J. Brooks, O. Toukolehto and M. Wren et al., 2013. Psychological trauma symptom improvement in veterans using emotional freedom techniques: A randomized controlled trial. J. Nervous Mental Dis., 201: 153-160. DOI: 10.1097/NMD.0b013e31827f6351

Clara, I.P., B.J. Cox and M.W. Enns, 2001. Confirmatory factor analysis of the depression anxiety stress scales in depressed and anxious patients. J. Psychopathol. Behav. Assessment, 23: 61-67. DOI: 10.1023/A:1011095624717

Craig, C., 2010. The Clinical EFT Manual. 1st Edn., Energy Psychology Press, Santa Rosa, California.

Craig, G. and A. Fowlie, 1995. Emotional freedom techniques. Self-published manual. The Sea Ranch.

Cuijpers, P., A.V. Straten and G. Andersson, 2008. Internet-administered cognitive behavior therapy for health problems: a systematic review. J. Behav. Med., 31: 169-177. DOI: 10.1007/s10865007-9144-1

Diepold, J.H. and D.M. Goldstein, 2008. Thought Field Therapy and QEEG changes in the treatment of trauma: A case study. Traumatology, 15: 85-93. DOI: $10.1177 / 1534765608325304$

Feinstein, D., 2008. Energy psychology: A review of the preliminary evidence. Psychotherapy: Theory. Res. Pract. Train., 45: 199-213. DOI: 10.1037/00333204.45.2.199

Feinstein, D., D. Eden and G. Craig, 2005. The Promise of Energy Psychology. 1st Edn., Tarcher. New York.

First, M.B., M. Gibbon, R.L. Spitzer, J.B.W. Williams and L.S. Benjamin, 1997. Structured Clinical Interview for DSM-IV Axis II Personality Disorders, (SCID-II). 1st Edn., American Psychiatric Press, Inc., Washington, D.C.

Forde, F., M. Frame, P. Hanlon, G. MacLean and D. Nolan et al., 2005. Optimum number of sessions for depression and anxiety. Nurs. Times, 101: 36-40. PMID: 16276843

Gallo, F.P., 2004. Energy Psychology: Explorations at the Interface of Energy, Cognition, Behavior and Health. 2nd Edn., CRC, New York.

Jones, S.J., J.A. Thornton and H.B. Andrews, 2011. Efficacy of Emotional Freedom Techniques (EFT) in reducing public speaking anxiety: A randomized controlled trial. Energy Psychol.: Theory Res. Treatment, 3: 19-32.
Karatzias, T., K. Power, K. Brown, T. McGoldrick and M. Begum et al., 2011. A controlled comparison of the effectiveness and efficiency of two psychological therapies for posttraumatic stress disorder: Eye movement desensitization and reprocessing Vs. emotional freedom techniques. J. Nervous Mental Dis., 199: 372-378. DOI: 10.1097/NMD.0b013e31821cd262

Kazdin, A.E. and L.A. Wilcoxon, 1976. Systematic desentisation and non specific treatment effects: A methodolical evaluation. Psychol. Bull., 83: 729758. DOI: 10.1037/0033-2909.83.5729

Kessler, R.C., J. Soukup, R.B. Davis, D.F. Foster and S.A. Wilkey et al., 2001. The use of complementary and alternative therapies to treat anxiety and depression in the United States. Am. J. Psychiatry, 158: 289-294. DOI: 10.1176/appi.ajp.158.2.289

Lovibond, P.F. and S.H. Lovibond, 1995. The structure of negative emotional states: Comparison of the major Depressive Disorder Anxiety Stress Scales (DASS) with the beck major depressive disorder and anxiety inventories. Behav. Res. Therapy, 33: 335-343. DOI: 10.1016/0005-7967(94)00075-u

Lovibond, P.F., D.A.T. Siddle, N.W. Bond, 1993. Resistance to extinction of fear-relevant stimuli: Preparedness or selective sensitization? J. Exp. Psychol., 122: 449-461. DOI: 10.1037/00963445.122.4.449

Marton, P., M. Korenblum, S. Kutcher, B. Stein and B. Kennedy et al. 1989. Personality dysfunction in depressed adolescents. Can. J. Psychiatry, 34: 810813. PMID: 2819648

Mollon, P., 2007. Thought field therapy and its derivatives: Rapid relief of mental health problems through tapping on the body. Primary Care Community Psychiatry, 12: 123-127. DOI: 10.1080/17468840701750836

Nader, K., G.E. Schafe and J.E. LeDoux, 2000. The labile nature of consolidation theory. Nature Neurosci. Rev., 1: 216-219. DOI: 10.1038/35044580

Osman, D., N. Buchon, S. Chakrabarti, Y.T. Huang and W.C. Su et al., 2012. Autocrine and paracrine unpaired signaling regulate intestinal stem cell maintenance and division. J. Cell Sci., 125: 5944-5949.DOI: $10.1242 /$ jcs. 113100

Rowe, J.E., 2005. The effects of EFT on long-term psychological symptoms. Counseling Clinical Psychol., 2: 104-111. 
Salas, M.M., A.J. Brooks and J.E. Rowe, 2011. The immediate effect of a brief energy psychology intervention (Emotional Freedom Techniques) on specific phobias: A pilot study. EXPLORE J. Sci. Heal., 7: 155-161. DOI: 10.1016/j.explore.2011.02.005.

Sezgin, N. and B. Özcan, 2009. The effect of progressive muscular relaxation and emotional freedom techniques on test anxiety in high school students: A randomized controlled trial. Energy Psychol. Theory, Res. Treatment, 1: 23-30. DOI: 10.9769.EPJ.2009.1.1.NS

Shedler, J., 2010. The efficacy of psychodynamic psychotherapy. Am. Psychol., 65: 98-109. DOI: 10.1037/a0018378

Sheehan, D.V., Y. Lecrubier, K.H. Sheehan, J. Janavs and E. Weiller et al., 1997. The validity of the Mini International Neuropsychiatric Interview (MINI) according to the SCID-P and its reliability. Eur. Psychiatry, 12: 232-241. DOI: 10.1016/S09249338(97)83297-X

Sheehan, D.V., Y. Lecrubier, K.H. Sheehan, P. Amorim and J. Janavs et al., 1998. The Mini-International Neuropsychiatric Interview (M.I.N.I.): The development and validation of a structured diagnostic psychiatric interview for DSM-IV and ICD-10.. J. Clin. Psychiatry, 59: 22-32. PMID: 9881538
Stapleton, P., T. Sheldon, B. Porter and J. Whitty, 2011. A randomised clinical trial of a meridian-based intervention for food cravings with a six-month follow-up. Behav. Change, 28: 1-16. DOI: 10.1375/bech.28.1.1

Strunk, D.R., M.A. Brotman, R.J. DeRubeis and S.D. Hollon, 2010. Therapist competence in cognitive therapy for major depressive disorder: predicting subsequent symptom change. J. Consult. Clin. Psychol., 78: 429-37. DOI: 10.1037/a0019631

Tolin, D.F., 2010. Is cognitive-behavioral therapy more effective than other therapies?: A metaanalytic review. Clinical Psychology Review, 30: 710-720. DOI: DOI: 10.1016/j.cpr.2010.05.003

Wells, S., K. Polglase, H.B. Andrews, P. Carrington and A.H. Baker, 2003. Evaluation of ameridianbasedintervention, Emotional Freedom Techniques (Clinical EFT), for reducing specific phobias of small animals. J. Clin. Psychol., 59: 943-966. DOI: $10.1002 /$ jclp.10189

WHO, 2004. Management of Mental Disorders. 4th Edn., WHO, Sydney.

WHO, 2008. The global burden of disease: 2004 update. WHO, Sydney.

Wolpe, J., 1958. Psychotherapy by Reciprocal Inhibition. 1st Edn., Stanford University Press, Stanford, ISBN-10: 0804705097. pp: 239.

Wolpe, J., 1969. The Practise of Behavioural Therapy. 1st Edn., Pergamon Press Ltd, New York. 